\title{
Small diameter blood vessel prostheses from blends of polyethylene oxide and polypropylene oxide
}

\author{
J.G.F. Bots, L. van der Does and A. Bantjes \\ Department of Chemical Technology, Biomaterials Section, Twente University of Technology, PO Box 217, 7500 AE Enschede. \\ The Netherlands \\ (Received 5 September 1985; accepted 1 November 1985)
}

\begin{abstract}
Studies on the relationship between blood platelet adhesion and type and amount of polyether segments in copolyetherurethanes report a reduced platelet adhesion with increasing polyether content. We therefore assumed that combinations of polyethylene oxide (PEO) and polypropylene oxide (PPOX) might give materials with a good blood compatibility. Water-soluble PEO was attached to PPOX by u.v.-initiated crosslinking. Films were tested for hydrophilicity, mechanical properties, protein adsorption and blood compatibility. The hydrophilicity was determined by swelling experiments. A compromise between hydrophilicity (PEO) and mechanical strength (PPOX) was met at a swelling of 0.5 (PPOX/PEO ratio: 90/10). In protein adsorption studies only small amounts of adsorbed proteins were found. Three blood material interaction in vitro tests gave good results: a low platelet adhesion and kallikrein generation and a high APTT value. Porous tubings (inner diameter $1.3 \mathrm{~mm}$ ) were fabricated, by spinning from solution, for implantation in the abdominal aorta of rats. Stress-strain diagrams were comparable to those reported for natural blood vessels.
\end{abstract}

Keywords: Prostheses. polyethers, blood vessels, in vitro evaluation. mechanical properties. polyethylene oxide, polypropylene oxide

A special type of block copolymer, consisting of alternating hard and soft segments, is widely known and two representatives, i.e. Biomer $r^{\oplus, 1}$ and Avcothane ${ }^{\infty}, 2$ are used as biomaterials in several blood contacting devices such as catheters, artificial blood vessels and heart valves. Kim et al. ${ }^{3}$ and Lyman et al. ${ }^{4}$ reported that a segmented copolyetherurethane-urea (PEUU) compared to silicone rubber (SR) and a fluorinated ethylene-propylene copolymer (FEP) showed an increased albumin adsorption together with a low $\gamma$-globulin and fibrinogen adsorption and a low platelet adhesion. This behaviour is generally accepted as being indicative of a blood material interaction without severe reactions like thrombus formation.

Indications were obtained by Lyman ${ }^{4}$ that protein is more strongly adsorbed onto the hard polyurethane-urea segments than on the soft polypropylene oxide blocks. He concluded from ESCA measurements that the air side of a cast film was richer in polyether than the glass mould side of the film.

Interesting observations have also been reported by Lelah et al. ${ }^{5}$, who found that extruded Biomer had a higher content of soft polytetramethylene oxide segments on the surface than cast Biomer $\$$, resulting in a lower fibrinogen adsorption and platelet adhesion for the former. Results from these and other studies ${ }^{6.7}$ indicate that the polyether segments in copolyurethanes are probably responsible for the preferred adsorption of albumin and the reduced platelet adhesion.

Apart from this, the ratio of hydrophilic to hydrophobic areas on the polymer surface is known to have a marked influence on the blood compatibility. For instance, a different behaviour in protein adsorption ${ }^{8}$ and platelet adhesion ${ }^{9.10}$ was found in contact with hydrophilic or hydrophobic surfaces.

The importance of the morphology and texture of the blood contacting surface has also been reported by Yui et al. ${ }^{11}$ and Hess et al. ${ }^{12}$. Yui synthesized polypropylene oxide segmented polyamides with various polyamide segment lengths and found the platelet adhesion to be strongly dependent on the size of the crystalline and amorphous domains. The platelet adhesion was significantly miminized on a copolymer, in which the average diameters of the crystalline and amorphous domains were 6.42 and $5.18 \mathrm{~nm}$ respectively. It was concluded from in vivo experiments ${ }^{12}$ that the neo-intima formation in small diameter $(1.5 \mathrm{~mm})$ prosthetic blood vessels was strongly stimulated by a porous fibrillar inner structure.

Sa da Costa ${ }^{13}$ extensively studied the relationship between platelet adhesion and type and amount of polyether segments in copolyetherurethanes and she found a reduced platelet adhesion with increasing polyether content. Polyethylene oxide (PEO) was reported to be most active in 
suppressing platelet adhesion compared to polypropylene oxide (PPOX) and polytetramethylene oxide (PTMO).

From these results we derived the hypothesis that combining PEO with PPOX or PTMO might lead to materials with a good blood compatibility: PEO as the blood compatible, hydrophilic component and PPOX or PTMO as the more hydrophobic and mechanically stronger one.

PEO was attached to high molecular weight atactic PPOX by u.v.-initiated crosslinking in the presence of dicumylperoxide (DCP). Swelling experiments were performed with the resulting films in order to determine the equilibrium water content. Blood material interactions were studied with three tests: (i) kallikrein generation (Factor XII activation), (ii) activated partial thromboplastin time, and (iii) blood platelet adhesion. Adsorption of albumin, fibrinogen, fibronectin and $\gamma$-globulin was investigated using an ELISA test ${ }^{8}$. For in vivo experiments blood vessel prostheses were spun from solution and implanted in the abdominal aorta of rats. The mechanical properties were compared to those of natural blood vessels.

\section{EXPERIMENTAL}

\section{Materials}

High molecular weight PEO, $\left(\overline{\mathrm{M}}_{\mathrm{w}}=1,3\right.$ and $\left.6 \times 10^{5}\right)$ was purchased from Polysciences Inc. (Warrington, USA). The crystalline and water-soluble polymers showed a broad molecular weight distribution: $\overline{\mathrm{M}}_{w} / \overline{\mathrm{M}}_{n}=6.1$ (GPC-LALLS).

Atactic PPOX (d.s.c.; $95 \%$ atactic) was synthesized ${ }^{14}$ from propylene oxide (Merck) with a catalyst prepared from diethylzinc [Schering AG, Berlin; 20\% (w/w) in toluene] and triphenyltin hydroxide ( $M \& T$, Vlissingen, The Netherlands). $\bar{M}_{w}$ varied between 0.8 and $2.5 \times 10^{5}$, depending on the monomer/catalyst ratio and for all PPOX batches $\overline{\mathbf{M}}_{w} / \overline{\mathrm{M}}_{\mathrm{n}}<1.3$. Semicrystalline PPOX was prepared ${ }^{15}$ with $\mathrm{FeCl}_{3}$ as a catalyst and the crystalline fraction (d.s.c.; $95 \%$ crystallinity) was separated from the amorphous one by recrystallization (twice) from methanol, which dissolved the atactic component.

Semicrystalline PTMO (d.s.c.; 70\% crystallinity) was synthesized from tetrahydrofuran (Merck) with $\mathrm{SbCl}_{5}$ as a catalyst ${ }^{16}$. Molecular weights of 2 to $4 \times 10^{4}\left(\overline{\mathrm{M}}_{w}\right)$ were obtained: $\bar{M}_{w} / \bar{M}_{n}=1.2$ (GPC-LALLS). For crosslinking experiments DCP (Schuchardt, Germany), recrystallized twice from methanol, was used. The solvents, used for the catalyst preparation for the polymerization of propylene oxide [(xylene, toluene and hexane) (Merck)] were distilled from sodium. Tetrahydrofuran and propylene oxide were purified by distillation from lithium aluminium hydride (Merck) and calcium chloride (Merck), respectively. All purifications were performed in a nitrogen atmosphere.

\section{Methods}

Film preparation and swelling experiments. Mixtures of PEO, PPOX and DCP were dissolved in dichloromethane or chloroform and films were cast from viscous $5-10 \%(w / w)$ solutions. After slow evaporation of the solvent the films were dried in vacuum $(0.2-0.5 \mathrm{mmHg})$ at room temperature and subsequently u.v.-irradiation (Hanau lamp TQ 81 , wavelengths 254 and $366 \mathrm{~nm}$ ) proceeded in a nitrogen atmosphere (the temperature during irradiation was kept constant). The irradiated films were washed with water for $24 \mathrm{~h}$ to remove non-crosslinked PEO and with acetone (24 h) for removal of non-crosslinked PPOX as well as DCP and decomposition products like acetophenone and 2-phenyl- isopropanol. Weight losses were determined and the equilibrium water content after $24 \mathrm{~h}$ of swelling was measured. Swelling was calculated using the relationship:

$$
S=W_{e q} W_{d r y}-1
$$

Films of PEO, PPOX and mixtures containing 4.8\% DCP $(w / w)$ for measurements of mechanical properties and protein adsorption were cast from $\mathrm{CH}_{2} \mathrm{Cl}_{2}$, irradiated for $1 \mathrm{~h}$ at $50^{\circ} \mathrm{C}$ and water washed (no acetone washing).

Samples for thermal analysis (d.s.c.) were cast from $\mathrm{CH}_{2} \mathrm{Cl}_{2}$ without adding DCP. The coatings of PPOX/PEO, PPOX, PTMO and Biome (Ethicon Inc., Sommerville NJ, USA) on the inside of glass tubes for the kallikrein generation and APTT test and on glass slides for the blood platelet adhesion tests were obtained by covering the glass surfaces with a thin layer of a slightly viscous solution of Biomer (DMF), PTMO (THF) or PPOX, PPOX $/$ PEO $\left(\mathrm{CH}_{2} \mathrm{Cl}_{2}\right)$ and allowing the solvent to evaporate. The PPOX containing coatings [with an initial DCP content of $4.8 \%(w / w)$ ] were irradiated for $1 \mathrm{~h}$ at $50^{\circ} \mathrm{C}$ and subsequently washed with water (no acetone washing since this loosens the coating).

Blood vesse/ prostheses. From a $4 \%(w / w)$ solution of PPOX/PEO/DCP: 85.8/9.5/4.7 (\%, w/w) in dichloromethane/dichloroethane: $70 / 30(\mathrm{v} / \mathrm{v})$ fibres with a diameter ranging from 5 to $50 \mu \mathrm{m}$ were spun and by winding on a rotating axis porous 'woven-like' tubings were fabricated (and subsequently u.v.-irradiated during $60 \mathrm{~min}$ ). Inner and outer diameter could be varied by changing the axis diameter $(0.6-2.0 \mathrm{~mm})$ and the spinning time $(30 \mathrm{~min}-2 \mathrm{~h})$. The pore size (5-200 $\mu \mathrm{m})$ as well as the fibre diameter could be varied depending on the flow rate of the polymer solution.

Mechanical properties. Stress-strain measurements were made with porous tubings (length $40 \mathrm{~mm}$; inner diam. $1.3 \mathrm{~mm}$; outer diam. $1.7 \mathrm{~mm}$ ) and dumbbell shaped test strips $(6 \times 40 \mathrm{~mm})$ using a tensile testing device (Instron floor model TT-CM, High Wycombe, UK) with drawing rates of $5 \mathrm{~mm} / \mathrm{min}$.

Differential scanning calorimetry. In the temperature range from $20-100^{\circ} \mathrm{C}$ thermograms were recorded using a Du Pont DSC 990 at a heating rate of $10^{\circ} \mathrm{C} / \mathrm{min}$. Indium was used to calibrate the temperature scale and to determine the heat of fusion of PEO, atactic and crystalline PPOX and PTMO. Transition temperatures were determined for PEO, PPOX and PTMO with samples weighing $10 \pm 2 \mathrm{mg}$.

Protein adsorption. Crosslinked films of PPOX/PEO: 90/10 were washed with PBS buffer and screened on adsorption of albumin (BSA), fibronectin, fibrinogen and $\gamma$-globulin with an ELISA test ${ }^{8}$. Equilibrium protein adsorption was measured after $100 \mathrm{~min}$ incubation with human plasma. Tissue culture polystyrene (Costar, Cambridge, Mass., USA) was taken as reference material and Biome ${ }^{\$}$, cast from DMF $[10 \%(w / w)$, air side] was used for comparison.

Kallikrein generation ${ }^{17}$. This could be detected using a chromogenic substrate (AB KABI Diagnostics, type S2302). The release of $p$-nitroaniline was measured by spectrophotometric analysis at $405 \mathrm{~nm}$. Aliquots of $1 \mathrm{ml} 10 \%$ citrated human plasma [diluted to $10 \mathrm{ml}$ with Tris buffer, $50 \mathrm{~mm}$, $\mathrm{pH} 7.4$ (Merck)] were incubated at $37^{\circ} \mathrm{C}$ in polymer coated glass tubes (see Film preparation). Within a period of $15 \mathrm{~min}$, at time $=1,2,3.5,5,7.5,10,12.5$ and $15 \mathrm{~min}$, samples of $100 \mu$ l were taken and added to the chromogenic 
substrate $(300 \mu \mathrm{l}$ Tris buffer and $100 \mu \mathrm{l}$ S2302 stock solution, $37^{\circ} \mathrm{C}$ ). After addition of $200 \mu \mathrm{l} 50 \%(\mathrm{v} / \mathrm{v})$ acetic acid solution the enzymatic reaction was stopped and the released $p$-nitroaniline was measured. The reference materials were silica borate glass and $S^{18}$ (RTV adhesive sealant, General Electric, Bergen op Zoom, The Netherlands), the latter as a coating on glass tubes [from a $5 \%(w / w)$ solution in THF: see Film preparation].

APTT test ${ }^{19}$. Citrated human plasma $(0.5 \mathrm{ml})$ was incubated at $37^{\circ} \mathrm{C}$ in polymer coated glass tubes. After $1 \mathrm{~min} 50 \mu \mathrm{l}$ cefalin solution [Sigma, RBC, $1 / 3$ stock suspension, $2 / 3$ $0.85 \%(\mathrm{w} / \mathrm{w}) \mathrm{NaCl}$ solution] was added, followed after another $1 \mathrm{~min}$ by $50 \mu \mathrm{l} 0.2 \mathrm{M} \mathrm{CaCl}_{2}$ solution (Merck). The interval between $\mathrm{CaCl}_{2}$ addition and the beginning of coagulation was determined (references: silica borate glass and SR).

Blood platelet adhesion ${ }^{20}$. A cell consisting of two glass slides, separated by a PVC ring, (inner diameter $10 \mathrm{~mm}$ ) was filled without air bubbles with citrated human plasma containing $\pm 5 \times 10^{4}$ platelets $/ \mu$ l. One of the glass slides was coated with the polymer to be tested (see Film preparation), the other with SR as a reference. The cell was centrifuged twice, the first time in a centrifugal force towards the polymer coated test surface and afterwards to the reference by turning the cell $180^{\circ}$. The adhered platelets on the polymer surfaces were counted using a microscope (magnification $\times 400$ ).

\section{RESULTS AND DISCUSSION}

In order to study the crosslinking process, films were prepared from PEO $\left(\overline{\mathrm{M}}_{\mathrm{w}}=6 \times 10^{5}\right)$ differing in initial $\mathrm{DCP}$ content and u.v.-irradiation time. Figure 1 shows a decrease of swelling $(S)$ with increasing DCP concentrations and

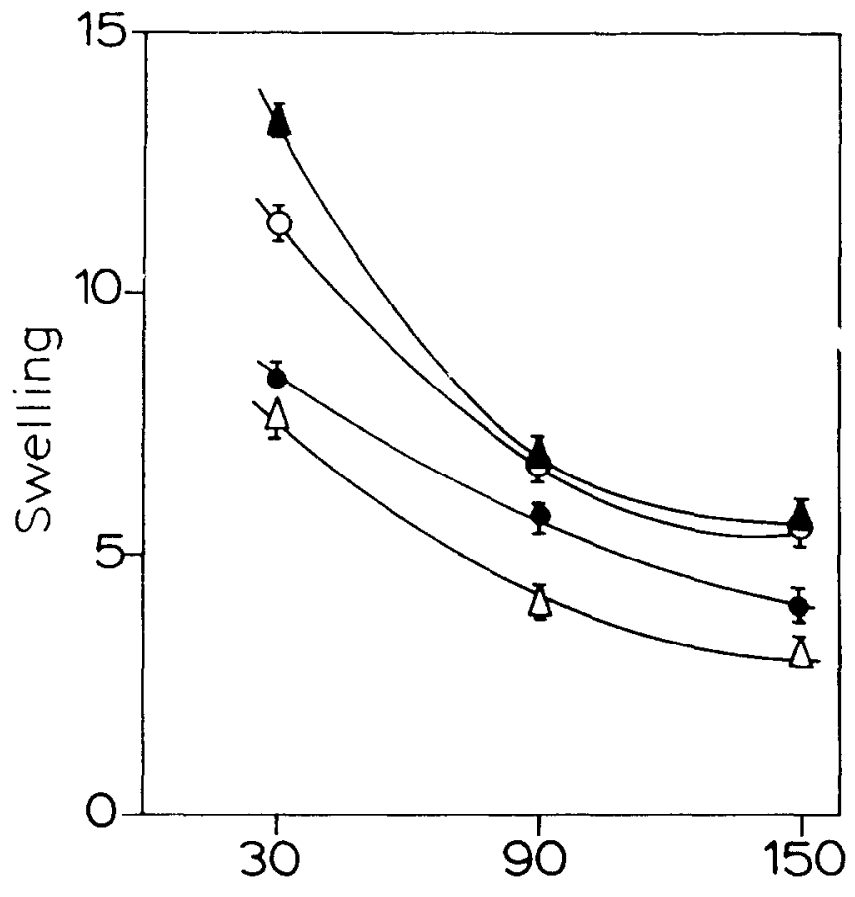

\section{U.V. - irradiation ( $\mathrm{min}$ )}

Figure 1 Swelling of PEO/DCP irradiated $\left(50^{\circ} \mathrm{C}\right)$ films. \% DCP $(w / w)$ : A. $4.8 ; 0,10.5 ; \bullet, 13.0 ; \triangle, 16.7$.
Table 1 Weight losses of PEO/DCP films in water and acetone

\begin{tabular}{rccc}
\hline u.v.-Irradiation (min) & DCP (\%) & \multicolumn{2}{c}{$\begin{array}{c}\text { Weight losses }(\%) \\
\text { Acetone }\end{array}$} \\
\hline 30 & & Water & 4 \\
90 & 4.8 & 55 & 3 \\
150 & 4.8 & 30 & 2 \\
30 & 4.8 & 20 & 14 \\
90 & 16.7 & 23 & 9 \\
150 & 16.7 & 13 & 6 \\
\hline
\end{tabular}

longer irradiation time. The increasing crosslink density lowers $S$ and the amount of noncrosslinked PEO, which could be determined by washing with water after irradiation (Tab/e 1). After washing with acetone in order to remove DCP and decomposition products it could be concluded from n.m.r. measurements of the acetone fraction that not all DCP had been used, - even after 150 min irradiation - , and that a certain amount of peroxy radicals was probably incorporated in the polymer network.

The temperature dependence of the curing process was investigated by irradiating $10 \% \mathrm{DCP}(\mathrm{w} / \mathrm{w})$ containing films at $20^{\circ}, 35^{\circ}, 50^{\circ}, 75^{\circ}$ and $90^{\circ} \mathrm{C}$ for 90 min with u.v. At $75^{\circ}$ and $90^{\circ} \mathrm{C}$ the polymer films deformed and melted (d.s.c.: $\mathrm{t}_{\mathrm{m}}=66^{\circ} \mathrm{C}$ ). The $50^{\circ} \mathrm{C}$ sample gave the highest crosslink density: $S=6.8$, whereas at $35^{\circ}$ and $20^{\circ} \mathrm{C} S$ values of 7.8 and 10 were found respectively, illustrating the influence of the temperature on the crosslinking process. The resulting films had no mechanical strength due to the high water content.

Attempts were made to reduce the swelling and to improve the mechanical properties by preparing films of PEO and PPOX in the presence of DCP. A linear relationship between the PEO content and $S$ was found for irradiation times of 30,90 and 150 min at $50^{\circ} \mathrm{C}$ and it is obvious from the data in Figure 2 that the presence of PPOX had resulted

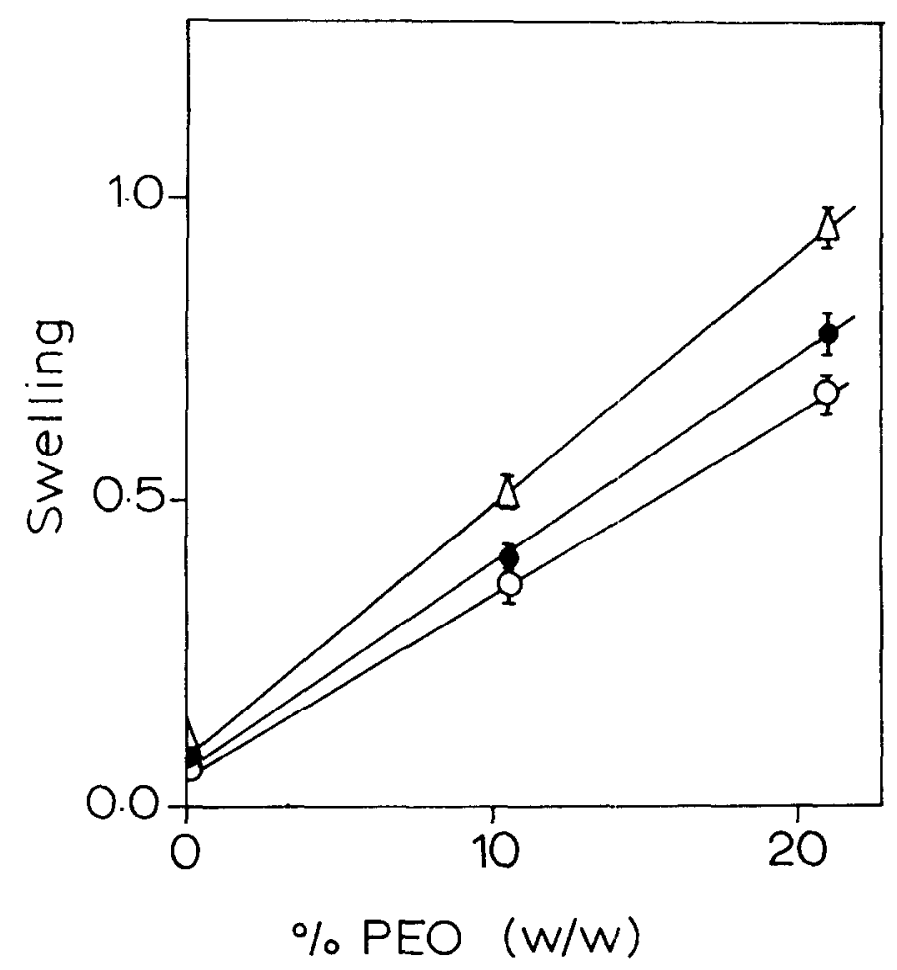

Figure 2 Swelling of PPOX/PEO irradiated $\left(50^{\circ} \mathrm{C}\right)$ films; $D C P: 9 \%(\mathrm{w} / \mathrm{w})$. u.v.-Irradiation (min): $\triangle, 30 ; 0,90 ; 0,150$. 
in a substantial decrease of the swelling. Weight losses of 15-25\% after washing with acetone, which dissolves PPOX, DCP and decomposition products, were observed but no relationship between weight loss and irradiation time could be determined. Extraction of the films with water to remove non-crosslinked PEO gave 0-5\% decrease in weight, indicating that nearly all of the PEO had been crosslinked.

Unlike the PEO/DCP samples the PPOX containing films showed elastomeric properties. However, for the preparation of these films a rather high DCP content and u.v. radiation times of 30-150 min were used. For possible application as biomaterials the concentration of the toxic DCP should be as low as possible and long u.v. radiation times should be avoided to prevent polymer degradation and incorporation of peroxy radicals in the polymer network. By lowering the DCP content the same $S$ values can be obtained by longer u.v.-irradiation. Films were made from PPOX/PEO having a low DCP content $[4.8 \%(w / w)]$. Although a short u.v.-irradiation $(30 \mathrm{~min}$ ) was applied, films were obtained with not too high $S$ values.

Stress-strain diagrams (Figure 3) of these films having a PPOX/PEO ratio of $100 / 0,90 / 10$ or $80 / 20$ (irradiated at $50^{\circ} \mathrm{C}$ ) show that the stress for a particular strain increases with an increase in PEO content. Figure 4 shows the stress-strain curves of PPOX and PPOX/PEO $(90 / 10)$ films before and after u.v.-irradiation. The behaviour of the irradiated films differs from the classical theory, which predicts an increase in stress at any particular strain due to crosslinking.

For the interpretation of the results of the blood material interaction tests information about the morphology of the polymeric films is necessary. It has been reported ${ }^{21}$ that the miscibility of PPOX and PEO is confined to the very low molecular weight polymers i.e. PPOX: $\bar{M}_{w}=425$ and PEO: $\bar{M}_{w}=300$. Mixtures of higher molecular weight polymers are incompatible and phase separation occurs. In the compatible mixtures Rastogi and St. Pierre ${ }^{22}$ observed

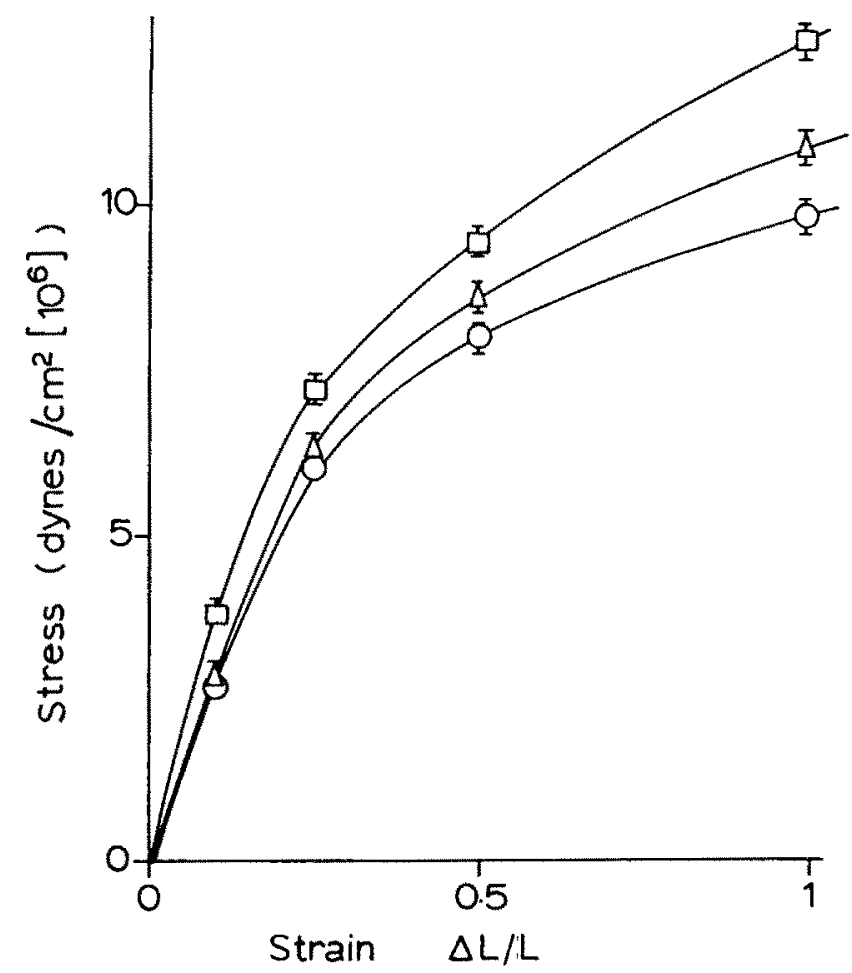

Figure 3 Stress-strain diagram of irradiated and water-washed PPOX/PEO films. O, PPOX; $\triangle$; PPOX/PEO, (90/10); [ ], PPOX/PEO (80/20).

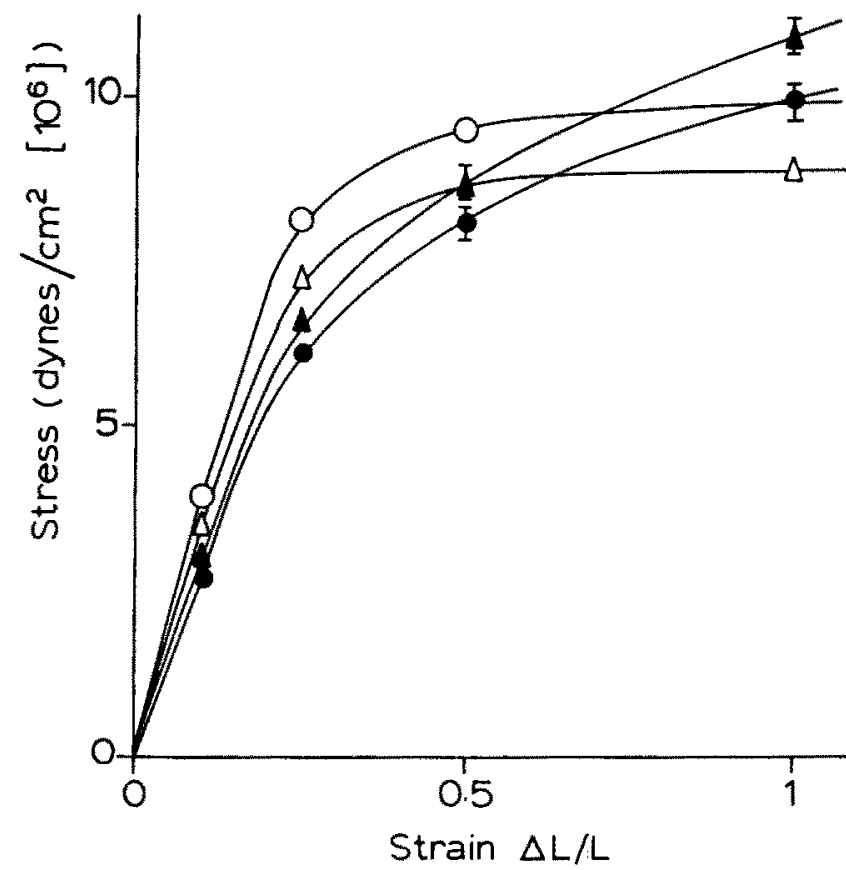

Figure 4 Stress-strain diagram of PPOX/PEO films; influence of the irradiation and water-washing. Open symbols = before irradiation; closed symbols = afterirradiation: O, PPOX; $\triangle$, PPOX/PEO (90/10);, PPOX/u.v.; A. PPOX/PEO $(90 / 10)$ u.v.

surface activity and for binary blends of PPOX and PEO this results in an increased surface concentration of PPOX. In order to get more information about the morphology of our high molecular PPOX/PEO blends, d.s.c. and ESCA studies are in progress.

Blood material interactions of the polymeric films as coatings on glass were studied with three in vitro tests. The materials were crosslinked films of PPOX and PPOX/PEO $(90 / 10)$ [containing $4.8 \%(w / w)$ and irradiated with u.v. for $1 \mathrm{~h}$ at $50^{\circ} \mathrm{C}$ ] and PTMO and Biomer for comparison. The PPOX/PEO $80 / 20$ film $(S=0.9)$ could not properly be attached to glass and has therefore not been tested. The in vitro tests were performed with coatings still containing DCP and/or decomposition products, because washing with acetone could not be applied owing to loosening of the coatings.

Figure 5 shows the results of the kallikrein generation test, normalized to silica borate glass as a strongly activating

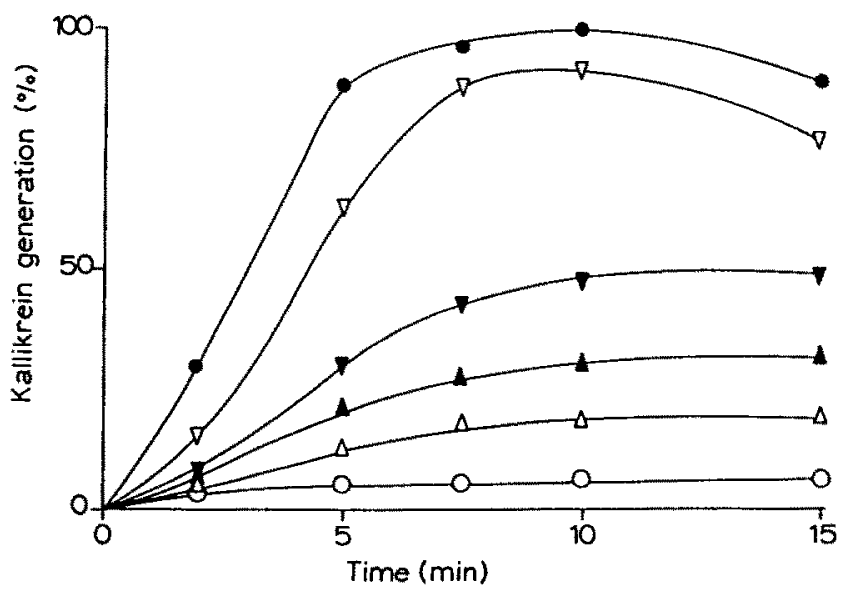

Figure 5 Kallikrein generation of PPOX/PEO (90/10), PPOX. PTMO. Biomer, SR and glass. $\triangle$ PPOX/PEO (9O/10); $\nabla$, PPOX; $\nabla$, PTMO; $\bullet$ glass, $\mathrm{O}, S R ; \mathbf{A}$, Biomer. 
surface and compared to silicone rubber, which is nonactivating. Kallikrein generation reflects the activation of Factor XII, which generally has been accepted to occur at negatively-charged surfaces. Although no net charge is present on the polyether surfaces, a generation of kallikrein was observed, possibly resulting from a certain degree of polarity in the carbon-oxygen bonds. In discussing kallikrein generation by the polyethers it should also be mentioned that PPOX coatings with and without DCP gave about the same results, indicating only a small effect of remaining DCP and/or decomposition products.

The polyethers studied differ in hydrophilicity and crystallinity, which might result in different Factor XII activation. The polymers PPOX and PTMO are both hydrophobic with $S$ values of 0.07 and 0.04, respectively (Sa da Costa found 0.05 for PTMO). From d.s.c. measurements it was concluded that PPOX was highly atactic, whereas PTMO had a crystallinity of about $70 \%$. Because of the comparable, slightly hydrophilic character, the difference in Factor XII activation may be attributed to the crystallinity of PTMO.

If crystallinity is assumed to stimulate Factor $\mathrm{XI}$ activation, the presence of crystalline PEO in PPOX/PEO $(90 / 10)$ would have been expected to result in an increase of kallikrein generation. Merrill et al. ${ }^{23}$ have reported, however, that equilibration of a copolyetherurethane with water at $25^{\circ} \mathrm{C}$ depressed the crystalline-amorphous transition of the soft PEO segments to temperatures below $0^{\circ} \mathrm{C}$, thus creating an 'amorphous' hydrophilic PEO phase.

The presence of PEO in PPOX/PEO (90/10), probably as an amorphous phase, had a marked effect on the hydrophilicity, with $S$ values of 0.3 to 0.5 depending on the irradiation time. The reduction of the kallikrein generation, compared to PPOX and PTMO, therefore seems to indicate a relationship between hydrophilicity and Factor XII activation.

The results of the APTT test (Table 2), displaying the activation of both the intrinsic and extrinsic coagulation system, show the same trend as the kallikrein generation, giving the highest coagulation time for PPOX/PEO $(90 / 10)$. Blood platelet adhesion tests (Tab/e 3) confirmed the findings of Lyman et $a .^{4}$ and Sa da Costa et al. ${ }^{6,7}$, giving the lowest platelet adhesion for PPOX/PEO (90/10).

From the results of the kallikrein generation test, the APTT determination and the platelet adhesion experiments it can be concluded that the in vitro blood compatibility of the polyethers, in particular of the PPOX/PEO $(90 / 10)$, is as good as or even better than of Biomer ${ }^{\boxplus}$.

Table 2 APTT values of polyethers, Biomer", SR and glass

\begin{tabular}{ll}
\hline Polymer & APTT (s) \\
\hline PTMO & $245 \pm 40$ \\
Biomer & $425+20$ \\
PPOX & $490 \pm 25$ \\
PPOX/PEO $(90 / 10)$ & $580 \pm 30$ \\
SR & $650 \pm 30$ \\
\hline Glass & $150 \pm 20$ \\
\hline
\end{tabular}

Table 3 Blood platelet adhesion on polvethers, Biomer and SR

\begin{tabular}{lc}
\hline Polymer & Platelets $/ 4 \times 10^{4} \mu \mathrm{m}^{2}$ \\
\hline PTMO & $90 \pm 20$ \\
Biomer & $120 \pm 30$ \\
PPOX & $12 \pm 4$ \\
PPOX $/$ PEO $(90 / 10)$ & $5 \pm 2$ \\
SR & $600 \pm 70$ \\
\hline
\end{tabular}

Table 4 Protein adsorption ${ }^{a}$ on polyethers, Biomers, and tissue culture polystyrene surfaces

\begin{tabular}{lcclc}
\hline Polymer & Albumin & Fibrinogen & Fibronectin & $\gamma$-globulin \\
\hline PPOX & $12 \pm 10$ & $15 \pm 12$ & $10 \pm 2$ & $19 \pm 15$ \\
PPOX/PEO $(90 / 10)$ & $8 \pm 6$ & $6 \pm 1$ & $13 \pm 1$ & $9 \pm 2$ \\
PPOX/PEO $(80 / 20)$ & $9 \pm 7$ & $6 \pm 1$ & $14 \pm 10$ & $9 \pm 7$ \\
Biomer & $450 \pm 10$ & $480 \pm 50$ & $10 \pm 10$ & $11 \pm 10$ \\
TCPS & $1900 \pm 400$ & & & \\
\hline
\end{tabular}

${ }^{\mathrm{a}} \mathrm{A}_{450} \times 10^{-3}$
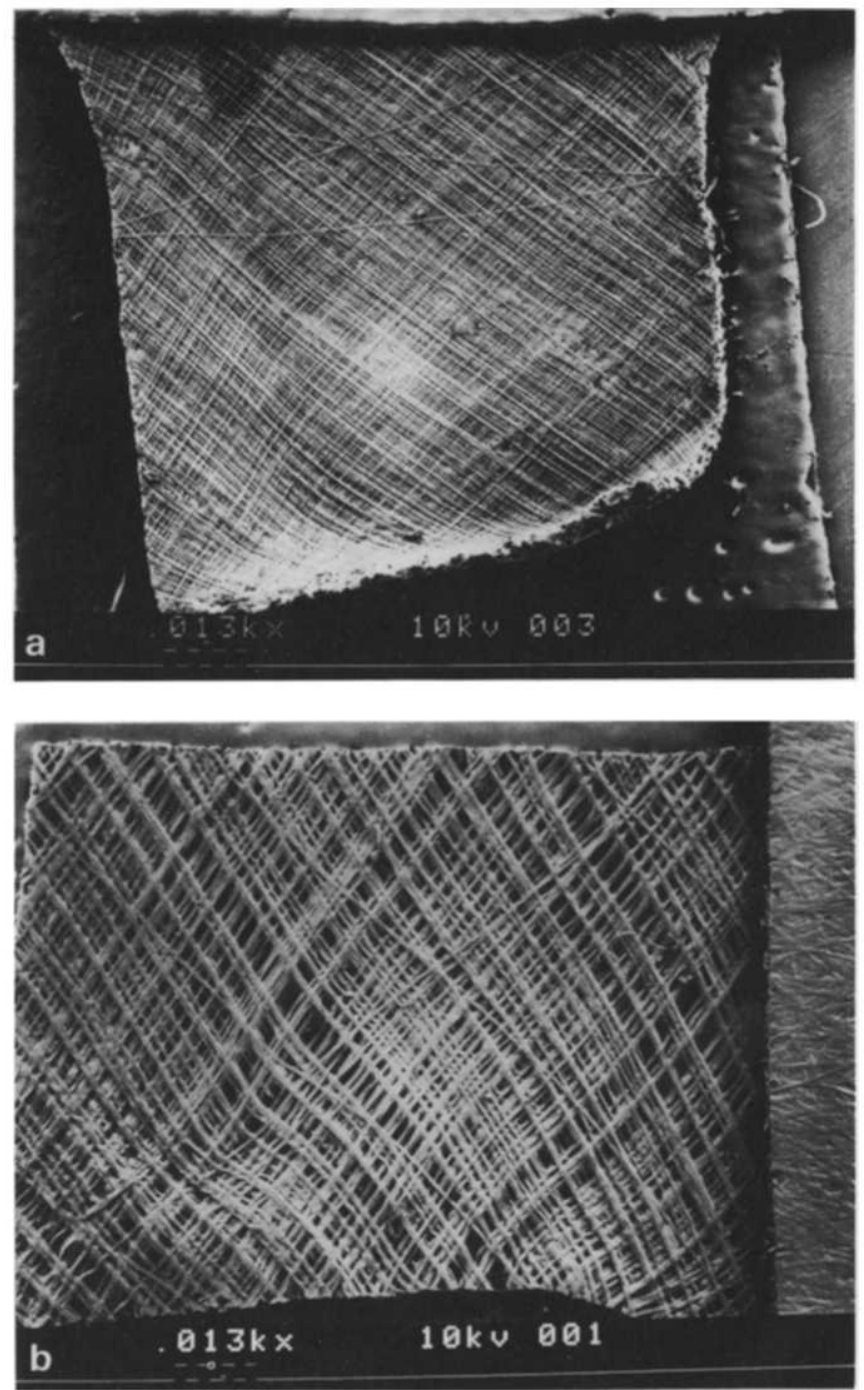

Figure 6 Lumen (a) and outside (b) of a prosthesis $(\times 10)$.

In order to elucidate the difference in behaviour between the polyethers and Biomer ${ }^{\$}$, in contact with blood or plasma, protein adsorption was studied using an ELISA test, in which absorbance is measured at $450 \mathrm{~nm}^{8}$. Fibrinogen deposition (Tab/e 4) was not significant on the polyether surfaces (PPOX/PEO: 100/0, 90/10 and $80 / 20$ ) in contrast to the adsorption on Biomer ${ }^{*}$, cast from DMF. Lelah et $a I^{5}$ found considerably less fibrinogen adsorption on the surface of extruded Biomer than on Biomer ${ }^{8}$, cast from DMA. Fibrinogen adsorption on the polyether surfaces is lower than on extruded Biomer which has been reported by Lelah et al. to be the most thromboresistant.

The very low protein adsorption on the polyethers suggests a certain inertness towards protein induced 

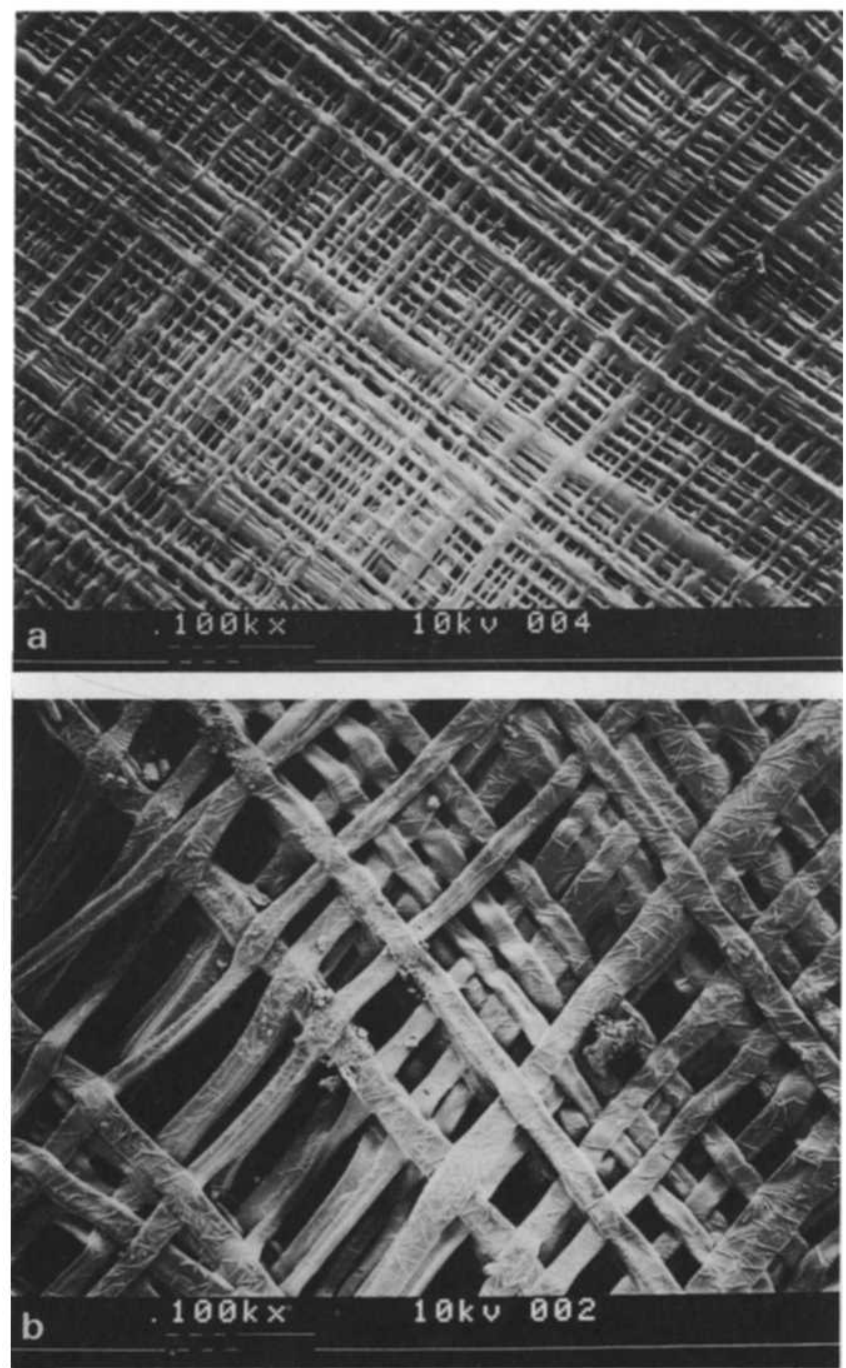

Figure 7 Inner (a) and outer (b) fibre morphology $(\times 80)$.

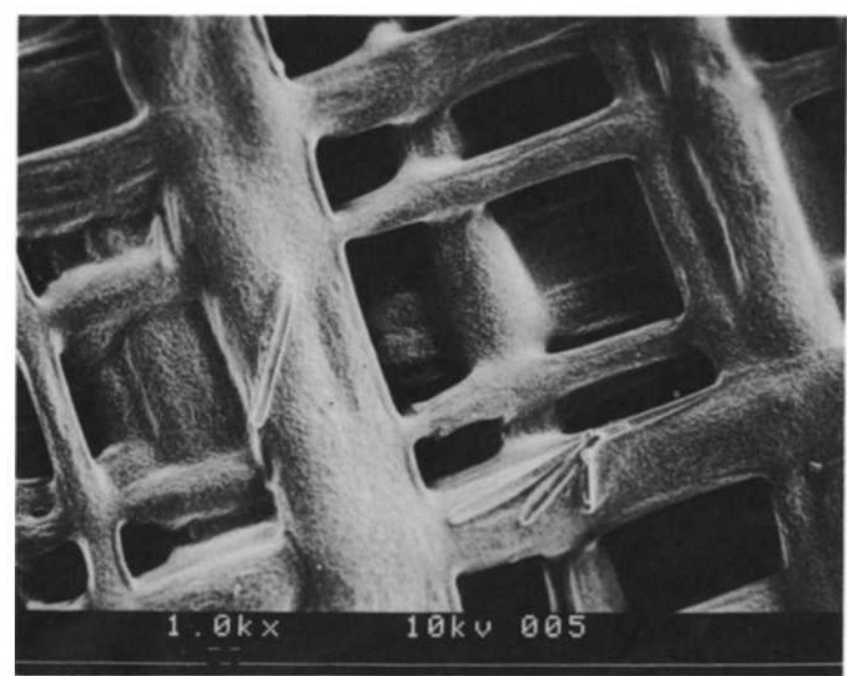

Figure 8 Confivency at fibre crossings $(\times 800)$

reactions like fibrin and thrombus formation, however, more detailed information has to be derived from in vivo experiments.

For this reason porous tubings were spun from solution [PPOX/PEO: 90/10; DCP: $4.8 \%(w / w), 1$ h u.v. at $50^{\circ} \mathrm{C}$. Histiopathological observations of White et al. ${ }^{24}$ on porous elastomeric polymer samples in dogs illustrate the influence of porosity on tissue ingrowth: small pores (18$45 \mu \mathrm{m}$ ) allow ingrowth of histiocytic tissue whereas larger
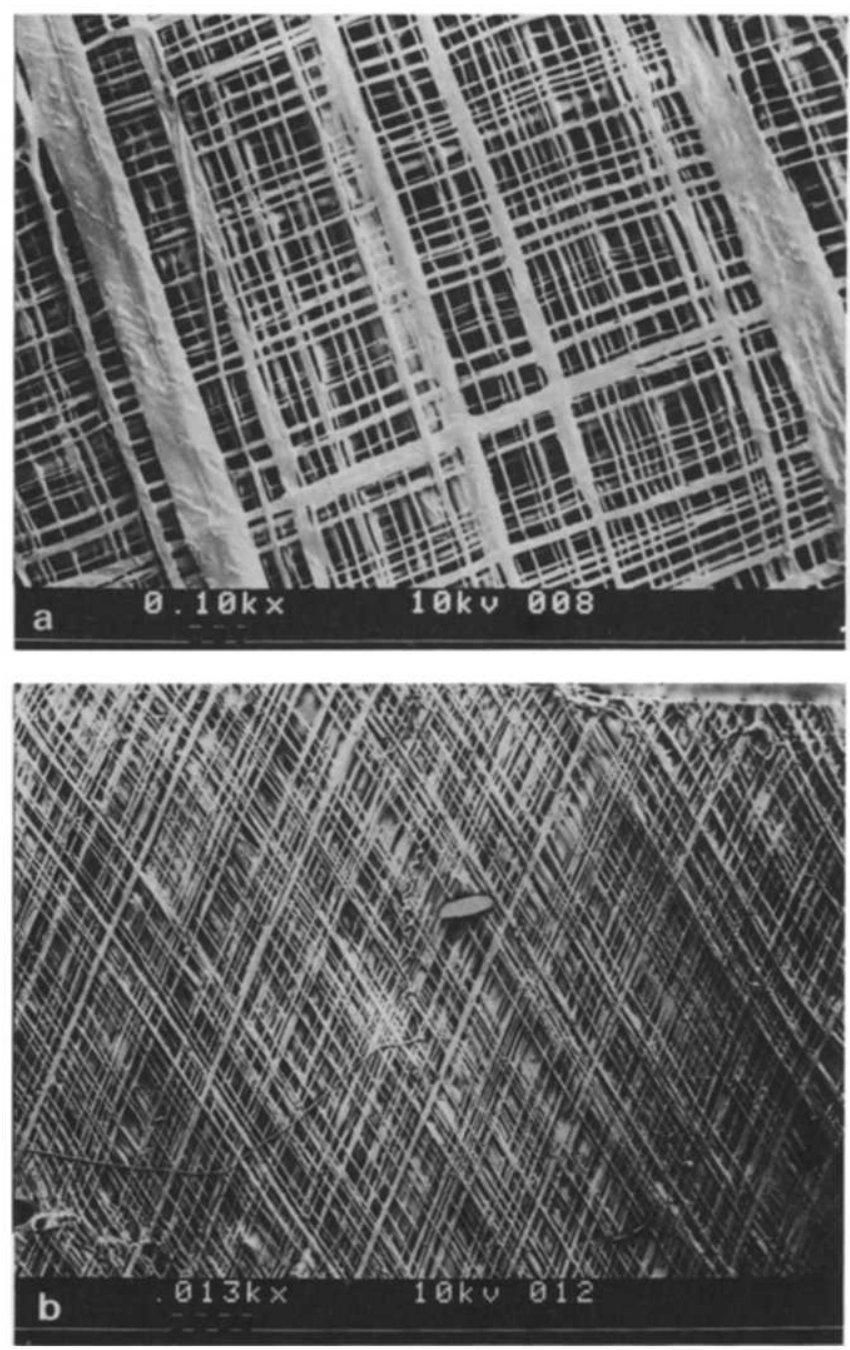

Figure 9 Winding angles of $45^{\circ} ; a_{1}(\times 86)$ and $60^{\circ} ; b_{1}(\times 10)$.

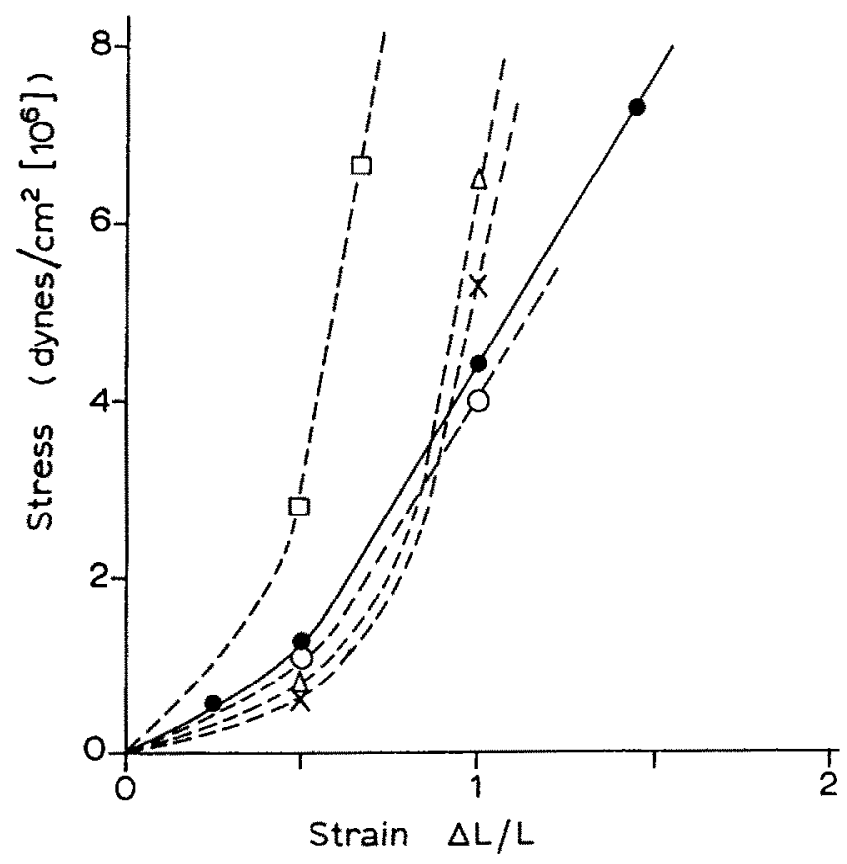

Figure 10 Longitudinal stress-strain diagram of PPOX/PEO; winding angfe $45^{\circ} . \square$ woven Dacron; $\triangle$, woven Tefion; 0 , femoral artery; $X$, abdominal aorta; ๑, PPOX/PEO (90/10).

pores $(60-120 \mu \mathrm{m})$ are ingrown with an organized, fibrous tissue, which provides more structural support to the 
implant than the former. Effects of the lack of pores in vascular grafts are reported by Lyman et al. ${ }^{25}$, who observed fibrotic reactions in solid wall Biomer grafts, also induced by the mismatch in mechanical properties between the prosthesis and the natural vessel.

Hess et al. ${ }^{12}$ found a continuous and permanent neointima on highly fibrillar surfaces with pore sizes of 20 $50 \mu \mathrm{m}$ and claimed that this structure provides essential anchoring facilities to the cells invading the prosthesis from the anastomotic areas.

From these experiments we designed a small diameter blood vessel prosthesis (inner diam. $1.3 \mathrm{~mm}$ ), having pores of 20-40 $\mu \mathrm{m}$ and fibres with diameters of 5-20 $\mu \mathrm{m}$ on the lumen side, which both gradually increase to pores of $100-$ $200 \mu \mathrm{m}$ and fibre diameters of 35-50 $\mu \mathrm{m}$ at the outside of the prosthesis. This concept allows formation of neo-intima beginning at the anastomoses using the anchoring positions of the fibrillar structure as well as ingrowth of tissue through the prosthesis wall to further support the endothelial layer.

Figure 6 shows the lumen and outside of the prosthesis at the same magnification, indicating the difference in pore diameter. A closer look (Figure 7) also illustrates the different fibre morphology. The large fibres are flattened after spinning due to the presence of a high boiling solvent (dichloroethane), which allows some deformation to take place but this is not observed for the smaller fibres, from which the solvent evaporates more easily. The presence of a high boiling solvent in the solvent mixture is necessary to obtain optimal confluency at the fibre crossings, which is essential for good mechanical properties like tear strength (Figure 8).

By changing the winding angle (Figure 9) the mechanical properties can be influenced. Stress-strain diagrams (Figure 10) of PPOX/PEO $(90 / 10)$ (winding angle $45^{\circ}$ ) indicate the resemblance between the prosthesis and the natural vessels $^{26}$ like the abdominal aorta and the femoral artery: the same low stress at small elongations, when predominantly changes in fibre orientation take place, is observed. Since for natural blood vessels the longitudinal distensibility tends to be less than the circumferential one, a winding angle of 35$40^{\circ}$ will result in optimal compliance in both directions, which according to Leidner et al. ${ }^{27}$ is advantageous since lower winding angles show less local fibre deformation caused by stress of sutures.

This study shows that by a simple procedure elastomeric products with a reproducible hydrophilicity can be made in combination with the spinning from solution method, which gives every opportunity to vary the pore size and fibre thickness of the prosthesis. This allows us to fabricate 'tailormade' small diameter blood vessel prostheses. The encouraging results from the in vitro tests and the good mechanical properties justify more research into the in vivo behaviour, which is performed at the moment with rats (abdominal aorta). The results of this in vivo study and the characterization of the blood contacting polyether surfaces with ESCA and d.s.c. will be published.

\section{ACKNOWLEDGEMENTS}

The authors are indebted to Dr Olijslager of KRI-TNO, Delft, for the blood platelet adhesion tests and to Dr Buijs of TNO, Utrecht, for assisting in the PPOX synthesis.
This study was subsidized by the Netherlands Organization for the Advancement of Pure Research (ZWO-SON).

\section{REFERENCES}

1 Ihlenfeld, J.V., Mathis, T.R., Riddle, L.M. and Cooper, S.L., Measurements of transient thrombus formation on polymeric materials Thromb. Res. 1979, 14, 953

2 Graham, S.W. and Hercules, D.M. Surface spectroscopic studies of Avcothane, J. Biomed. Mater. Res. 1981, 15, 349

3 Kim, S.W., Lee, R.G., Oster, H., Coleman, D., Andrade, J.D., Lentz, D.J. and Olsen, D., Platelet adhesion to polymer surfaces, Trans. Am. Soc. Artif. Int. Org. 1974. 20. 449

4 Lyman, D.J., Knutson, K, McNeill, B. and Shibatani, K., The effect of chemical structure and surface properties of synthetic polymers on the coagulation of blood, Trans. Am. Soc. Artif. Int. Org. 1975, 21, 49

5 Lelah, M.D., Lambrecht, L.K., Young, B.R. and Cooper, S.L.., Physicochemical characterisation and in vivo blood tolerability of cast and extruded Biomer, J. Biomed. Mater. Res. 1983, 17, 1

6 Sa Da Costa, V., Brier-Russell, D., Salzman, E.W. and Merrill. E.W. ESCA studies of polyurethanes, $J$. Coll. Interf. Sci. 1981, 80, 445

7 Sa Da Costa, V., Brier-Russell, D., Trudel III, G., Waugh, D.F., Saizman E.W. and Merrill, E.W., Polyetherurethane surfaces: thrombin adsorption, platelet adhesion and ESCA scanning. J. Coll. Interf. Sci. 1980, 76. 596

8 Breemhaar, W., Brinkman, E., Ellens, D.J., Beugeling, T. and Bantjes, A. Preferential adsorption of high density lipoprotein from blow plasma onto biomaterial surfaces, Biomaterials 1984, 5, 269

9 Ykada, Y., Iwata, H., Horii, F., Matsunaga, T., Taniguchi, M. Suzuki, M. Taki, W., Yamagata, S., Yonekawa, Y. and Handa, H., Blood compatibility of hycrophilic polymers, J. Biomed. Mater. Res., 1981, 15, 697

10 Coleman, D.L., Gregonis, D.E. and Andrade, J.D., Blood-materials interaction, J. Biomed. Mater. Res. 1982, 16, 381

11 Yui, N., Tanaka, J., Sanui, K, Ogata, N., Okano, T. and Sakurai, Y. Characterisation of the microstructure of polypropylene oxide segmented polyamide and its suppression of platelet adhesion, Polym. J. 1984, 16, 119

12 Hess, F., Jerusalem, C. Grande, P. and Braun, B, Significance of the inner surface structure of small caliber prosthetic blood vessels in relation to development, presence and fate of a neo-intima: A morphological evaluation, J. Biomed. Mater. Res. 1984, 18, 745

13 Sa Da Costa, V., PhD Thesis, Mass. Inst. Techn., USA, 1979

14 Buijs, H.C.W.M., US Patent No. 3798249 (1974)

15 Sorenson, W.R. and Campbell. T.W. Preparative Methods of Polymer Chemistry, Interscience, New York, 1968

16 Muetterties, E.L., US Patent Nos 2748145 (1956) and 2856370 (1958)

17 Hennink, W.E., Albumin-heparin conjugate as a coating for biomaterials, PhD Thesis, Techn. Univ. Twente, The Netherlands, 1985

18 Gallimore, M.J. and Friberger, P., Simple chromogenic peptide substrate assays for determining prekallikrein, kallikrein inhibition and kallikrein-like activity in human plasma. Thromb. Res. 1982, 25 293

19 Sederel, L., On the properties of a synthatic heparinoid polyelectrolyte and its biomedical applications, PhD Thesis. Techn. Univ. Twente. The Netherlands, 1982

20 George. J.M. Direct assessment of platelet adhesion to glass, Blood $1972,40,862$

21 Krause, S., Polymer-polymer compatibility, in Polymer Blends, (Eds D.R. Paul and S. Newman), Academic Press, New York, 1978

22 Rastogi, K. and St. Pierre, L.E., Interfacial phenomena in macromolecular systems III and V.J. Coll. Interf. Sci. 1969, 31, 168 and $1971,35,16$

23 Merrill. E.W., Salzman, E.W., Wan, S., Nahmud, N., Kushner, L., Lindon, J.N. and Curme, J., Platelet compatible hydrophilic segmented polyurethanes from polyethylene glycols and cyclohexane disocyanate, Trans. Am. Soc. Artit. Int. Org. 1982, 28, 482

24 White, R.A. Hirose, F.M., Sproat, R.W., Lawrence, R.S. and Nelson. R.J Histiopathologic observations after short term implantation of two porous elastomers in dogs, Biomaterials. 1981, 2, 171

25 Lyman, D.J.r Fazzio, F.J., Voorhees, H., Robinson, G. and Albo, D. Compliance as a factor effecting the patency of a copolyurethane vascular graft. J. Biomed. Mater. Res. 1978, 12, 337

26 Hasegawa, M. and Azuma, T., Mechanical properties of synthetic arterial grafts, J. Biomechan. $1978,12,509$

27 Leidner, J., Wong, E.W.C., MacGregor, D.C. and Wilson, G.J., A nove process for the manufacturing of porous grafts, $J$. Biomed. Mater Res. 1983, 17, 229 